\title{
Looking through the cracks of diabetic candidal balanoposthitis!
}

\author{
This article was published in the following Dove Press journal: \\ International Journal of General Medicine \\ 8 July 2011 \\ Number of times this article has been viewed
}

\author{
Shyam B Verma' \\ Uwe Wollina ${ }^{2}$ \\ 'Nirwana Clinic, Baroda, Gujarat, \\ India; ${ }^{2}$ Academic Teaching Hospital \\ Dresden-Friedrichstadt, Department \\ of Dermatology and Allergology, \\ Friedrichstrasse 4I, Dresden, \\ Germany
}

\begin{abstract}
India is becoming an epicentre of type II diabetes mellitus with a crude prevalence rate of about $9 \%$. Candida balanoposthitis is a known feature of diabetes mellitus especially in Indian males who are predominantly uncircumcised. In this country, diabetes is often diagnosed for the first time by dermatologists. Diabetes is much more frequently the cause of candida balanoposthitis than sexual intercourse in India. Fissuring along with balanoposthitis was found to be more common in sexually active males. The biomechanical basis of fissuring and the effect of diabetes in this phenomenon are explained. The issue of circumcision is debated under various aspects.
\end{abstract}

Keywords: balanoposthitis, diabetes mellitus, dermatology

Diabetes mellitus (DM) is a metabolic disease with a marked increase in prevalence not only in the western world but also in India. The current crude prevalence rate is said to be around 9\% in India. DM interferes with both facets of host defence against infection, the innate as well as the adaptive immunity. ${ }^{1-4}$

Acquired balanoposthitis can be the first clinical sign of DM in uncircumcised males. In a recent study from Britain, $26 \%$ of adult patients with an acquired phimosis were found to suffer from type II DM. The diagnosis of DM was made for the first time in $8 \%$ of these patients which means that candidal balanoposthitis in an apparently healthy male is a cutaneous marker of DM. Another $15 \%$ of males had impaired glycemic control. ${ }^{5}$ In a recent study from Portugal, prevalence of candida colonization was $26.2 \%$ and the prevalence of candida balanitis was $18 \%{ }^{6}$

We did an Internet-based survey of 20 dermatologists from across the country, who together cared for more than 60,000 dermatological outpatients. These dermatologists detected diabetes for the first time in $31 \%$ of patients presenting with candidal balanoposthitis. Their average figure of known diabetic patients coming with candidal balanoposthitis was 55\%. Figures in this clinic were on average $40 \%$ for the first time diagnosis of DM. About $75 \%$ of patients of candidal balanoposthitis seen in this clinic were known cases of DM. A large number of those who were sexually active had a tendency of fissuring of the inner part of the foreskin (Figure 1).

Those patients who were elderly and were not sexually active presented more commonly with itchy, moist erythema of the inner prepuce with variable involvement of the glans and significantly less fissuring. Itching, burning, and increased smegma collection were accompanying signs in the majority of young sexually active patients. (a)

Nirwana Clinic, Baroda, Gujarat, India

Tel +9l 2652322828

Fax +91 2652344137

Email skindinaverma@googlemail.com 


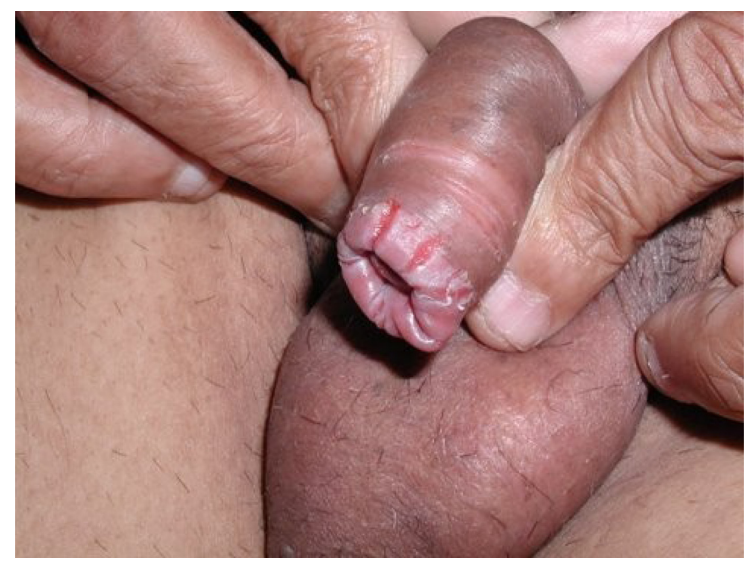

Figure I Fissuring in diabetes-related candida balanitis.

Candida balanoposthitis is a particularly important issue in countries like India where DM is emerging as a major public health problem, described by some as a pandemic. ${ }^{7}$ Lack of diagnosis or late diagnosis, irregular monitoring of blood sugar, and infrequent follow-up by the physician are also contributory. Diabetes caused candida balanoposthitis is more of a problem in India than sexually acquired candida balanoposthitis. Though no studies have been done to detect vulvovaginal candidiasis in the spouses of these men, a majority of them replied in negative when asked about an itchy, curdy, whitish vaginal discharge.

Religious beliefs also play an important part in the discussion of preventive measures. An overwhelming majority of Indian Hindu males are uncircumcised in contrast for instance to the Indian Moslem population but there are no scientific data available from India that demonstrate an advantage of circumcision in the prevention of candida balanoposthitis.

We realise that there is a paucity of dermatologic literature readily available explaining the phenomenon of fissuring in DM related balanoposthitis. It is important to bear in mind that balanoposthitis is a biomechanical problem, a fact that has become obscured in the discussion of the role of infections. Preputial fissures, a hallmark of this condition can be explained by the accumulation of advanced glycation end products (AGEs) in the skin. AGEs content is increased in particular by inadequate glycemic control in diabetics. AGEs impair production of collagen and extracellular organization that is associated with a lowered hydroxyproline content and superoxide dismutase activity. In addition, this causes deleterious effects such as a greater impairment and alteration of biomechanical properties of the skin, namely, elasticity and hydration. ${ }^{8-11}$ There is also loss of surface lipids of skin due to impairment of sebaceous gland function and a tendency of reduced hydration in the stratum corneum. ${ }^{9,12}$

Biomechanical stress due to the oft repeated acts of retracting the foreskin during urination and during sexual intercourse can directly be responsible for a tendency of vertical fissuring of the foreskin in diabetic patients. This can lead to fibrosis in the form of phimosis. ${ }^{6}$ It exacerbates candida balanoposthitis and hence the fissuring. Candida posthitis can develop without candida balanitis. It is of interest that an increased smegma production might be an attempt to control Candida overgrowth. It has been shown that lysozyme from prostate and seminal vesicles, which is present in smegma, is capable of inhibiting and eliminating Candida species. ${ }^{13}$ This would suggest that an intact prepuce would be protective, rather than an independent risk factor for candidal balanoposthitis. Surgery is in general not a cure for yeast infections with the exception of a possible abscess formation. $^{14}$

\section{Disclosure}

The authors report no conflicts of interest in this work. There was no funding.

\section{References}

1. Peleg AY, Weerathna T, McCarthy JS, Davis TME. Common infections in diabetes: pathogenesis, management and relationship to glycaemic control. Diabetes Metab Res Rev. 2007;23:3-13.

2. Moutschen MP, Scheen AJ, Lefebvre PJ. Impaired immune responses in diabetes mellitus: analysis of the factors and mechanisms involved. Relevance to the increased susceptibility of diabetic patients to specific infections. Diabetes Metab. 1992;18:187-203.

3. Belazi M, Velegraki A, Fleva A, et al. Candidal overgrowth in diabetic patients: potential predisposing factors. Mycoses. 2005;48: 192-196.

4. Hosteter MK. Effects of hyperglycaemia on $\mathrm{C} 3$ and Candida albicans. Diabetes. 1990;39:271-275.

5. Bromage SJ, Crump A, Pearce I. Phimosis as a presenting feature of diabetes. BJU Int. 2008;101:338-340.

6. Lisboa C, Santos A, Dias C, Azevedo F, Pina-Vaz C, Rodrigues A. Candida balanitis: risk factors. J Eur Acad Dermatol Venereol. 2010. In press.

7. Mohan V. Why are Indians more prone to diabetes. J Assoc Physicians India. 2004;52;468-474.

8. Ye X, Tong Z, Dang Y, et al. Effects of blood glucose fluctuation on skin biophysical properties, structure and antioxidant status in an animal model. Clin Exp Dermatol. 2009;35:78-82.

9. Seirafi H, Farsinejad K, Firooz A, et al. Biophysical characteristics of skin in diabetes: a controlled study. J Eur Acad Dermatol Venereol. 2008;23:146-149.

10. Yoon HS, Baik SH, Oh CH. Quantitative measurement of desquamation and skin elasticity in diabetic patients. Skin Res Technol. 2002;8:250-254.

11. Reihsner R, Melling M, Pfeifer W, Menzel EJ. Alterations of biochemical and two-dimensional biomechanical properties of human skin in diabetes mellitus as compared to effects of in vitro non-enzymatic glycation. Clin Biomech (Bristol, Avon). 2000;15:379-386. 
12. Sakai S, Kikuchi K, Satoh J, Tagami H, Inoue S. Functional properties of the stratum corneum in patients with diabetes mellitus: similarities to senile xerosis. Br J Dermatol. 2005;153:319-323.

13. Tobgi RS, Samaranayake LP, MacFarlane TW. In vitro susceptibility of Candida species to lysozyme. Oral Microbiol Immunol. 1988;3:35-39.
14. Davidson F. Yeasts and circumcision in the male. Br J Vener Dis. 1977;53:121-122.

International Journal of General Medicine

\section{Publish your work in this journal}

The International Journal of General Medicine is an international, peer-reviewed open-access journal that focuses on general and internal medicine, pathogenesis, epidemiology, diagnosis, monitoring and treatment protocols. The journal is characterized by the rapid reporting of reviews, original research and clinical studies across all disease areas.
A key focus is the elucidation of disease processes and management protocols resulting in improved outcomes for the patient.The manuscript management system is completely online and includes a very quick and fair peer-review system. Visit http://www.dovepress.com/ testimonials.php to read real quotes from published authors.

Submit your manuscript here: http://www.dovepress.com/international-journal-of-general-medicine-journal 